\title{
Modeling pegylated liposomal doxorubicin- induced hand-foot syndrome and intestinal mucositis in zebrafish
}

This article was published in the following Dove Press journal:

OncoTargets and Therapy

I July 2014

Number of times this article has been viewed

\author{
Yau-Hung Chen' \\ Ya-Ting Lee' \\ Chi-Chung Wen ${ }^{2}$ \\ Yun-Chen $\mathrm{Chen}^{3}$ \\ Yu-Jen Chen ${ }^{4,5}$ \\ 'Department of Chemistry, \\ ${ }^{2}$ Department of Mathematics, \\ ${ }^{3}$ Department of Chemical and \\ Materials Engineering, Tamkang \\ University, New Taipei City, Taiwan; \\ ${ }^{4}$ Graduate Institute of Pharmacology, \\ Taipei Medical University, Taipei, \\ Taiwan; ${ }^{5}$ Department of Radiation \\ Oncology, Mackay Memorial \\ Hospital, Taipei, Taiwan
}

\begin{abstract}
Pegylated liposomal doxorubicin (PLD) has been widely used to treat cancer. The adverse effects of PLD noted in clinical practice, especially hand-foot syndrome (HFS), are regarded as unique, and the management methods for them remain limited. This study was aimed at developing a feasible experimental model for translational medicine to solve this clinical issue by using skin fluorescent transgenic zebrafish. We established an optimal protocol for the administration of Lipo-Dox ${ }^{\mathrm{TM}}$, a PLD in current clinical use, to the $\operatorname{Tg}(k 18: d s r e d)$ zebrafish line expressing red fluorescence in keratinocytes. We made use of bodyweight, survival rate, gross observation, flssuorescent microscopic assessment, and pathological examination of the zebrafish to assess this model. The consecutive administration protocol of PLD resulted in growth retardation of the zebrafish embryo and survival impairment, indicating establishment of a significant toxicity. We observed fin necrosis and keratinocyte dissociation phenotypes in the PLD-treated fish after consecutive administration. The skin toxicity induced by the LipoDox injection was subsequently reversible, which might be compatible with a clinical course of skin recovery after discontinuation of Lipo-Dox administration. Furthermore, we found that the number of intestinal goblet cells, an important marker of intestinal inflammation, in the LipoDox-injected zebrafish was markedly increased, accompanied by impaired mucosal integrity. The intestinal inflammation induced by Lipo-Dox resembled the intestinal mucositis the clinical patients suffered from after the administration of PLD. In conclusion, we established a zebrafish model for PLD-induced HFS. The intestinal mucositis simultaneously noted in the PLD-treated zebrafish validated the similarity of clinical courses after administration of PLD. This model is easily assessable, efficient, and worthy for use in developing a new therapeutic protocol for prevention or treatment of HFS as well as intestinal mucositis. Further clinical investigations to validate the correlation between human and zebrafish data are warranted.
\end{abstract}

Keywords: doxorubicin, keratin, liposome, mucositis, skin, zebrafish

\section{Introduction}

Pegylated liposomal doxorubicin (PLD) has been widely used in the treatment of various types of cancers including ovarian, ${ }^{1}$ breast, ${ }^{2}$ and acquired immunodeficiency syndrome (AIDS)-related Kaposi's sarcoma. ${ }^{3}$ A Phase III trial for patients with platinum-sensitive relapsed/recurrent ovarian cancer demonstrated superiority in progression-free survival and a better therapeutic index for a combination of PLD with carboplatin in comparison with standard paclitaxel and carboplatin. ${ }^{4}$

PLD nanoparticles are doxorubicin hydrochloride encapsulated in pegylated liposomes. PLD is composed of distearoyl phosphatidylcholine and formulated with surface-bound methoxy polyethylene glycol, as for pegylation, to protect liposomes from detection by the mononuclear phagocytes and to increase blood circulation time. ${ }^{5}$ 
PLD displays linear pharmacokinetics over the dose range of $10-20 \mathrm{mg} / \mathrm{m}^{2}$ and nonlinear at a dose of $50 \mathrm{mg} / \mathrm{m}^{2}$. The plasma clearance of PLD is slow, and the area under the curve is approximately two to three orders of magnitude larger than a similar dose of free-form doxorubicin. ${ }^{6}$ The incidence of cardiotoxicity may be lower after PLD than after equivalent doses of standard doxorubicin. ${ }^{7}$

Among the adverse effects of PLD, hand-foot syndrome (HFS, also known as "palmar-plantar erythrodysesthesia") and intestinal mucositis ${ }^{8-10}$ often cause infection or severe pain, and may induce lethal gastrointestinal bleeding. HFS, especially, is the most common side effect which compromises patients' quality of life. The typical pathological changes of HFS include reddening, swelling, and desquamation on palms and soles. ${ }^{11}$ To date, the known protocols for prevention or treatment of HFS are limited except for one protocol describing that cooling the hands and feet during PLD therapy may help relieve the HFS symptoms. ${ }^{12}$ In this regard, establishing an animal model of HFS is an important step for developing agents to prevent or treat HFS.

The zebrafish has been accepted as a feasible model for toxicological study. ${ }^{13}$ There are abundant fluorescent zebrafish lines available to detect and observe subtle changes on tissue level grossly. ${ }^{14,15}$ For example, a green fluorescent kidney [Tg(wt1b:GFP)] and a red fluorescent skin transgenic zebrafish line $[\operatorname{Tg}(k 18: d s r e d)]$ have been reported to evaluate the toxic effects on kidney and skin. ${ }^{15,16}$ This advantage makes observation for phenotypic alterations of target tissues convenient and specific.

In this study, we established a zebrafish model for PLD-induced HFS and intestinal mucositis. The gross feature with quantitation and the pathological changes in skin fluorescent transgenic zebrafish were demonstrated.

\section{Materials and methods}

\section{Zebrafish}

To investigate the adverse effects of PLD in a zebrafish model, we obtained a skin fluorescent transgenic zebrafish line established by Professor HJ Tsai, ${ }^{17,18} \mathrm{Tg}(k 18: d s r e d)$, from Tsai's lab and raised them to $0.1-0.2 \mathrm{~g}$ (around 35-42 days post-fertilization) before the experiment.

\section{Chemicals and PLD administration}

The PLD used in this study, Lipo-Dox ${ }^{\mathrm{TM}}(20 \mathrm{mg} / 10 \mathrm{~mL})$, was purchased from TTY Biopharm (Taipei, Taiwan). For drug treatment, the $\operatorname{Tg}(k 18: d s r e d)$ zebrafish were dosed (by intraperitoneal injection) either without Lipo-Dox (buffer only; mock-control group, $n=6$ ) or with a buffer containing $40 \mu \mathrm{g} / \mathrm{g}$ of Lipo-Dox (Lipo-Dox-injected group, $[\mathrm{n}=12, \# 1-\# 12])$. The injection protocol is summarized in Figure 1. In brief, we injected Lipo-Dox one dose $(40 \mu \mathrm{g} / \mathrm{g})$ at day 0 , and subsequently injected one dose per week for 4 weeks (days 7, 14, 21, and 28), and examined the zebrafish phenotypic defects at each check point (days 10, 17, 24, 31, 38,45 , and 51).

\section{Gross and microscopic assessment of phenotype}

All of the fish samples were observed under a microscope (DM 2500, Leica; Buffalo Grove, NY, USA) equipped with a fluorescent DsRed filter cube (Kramer Scientific, Amesbury, MA, USA), and we captured pictures of the fish at particular stages using a digital camera (Sony, Tokyo, Japan).

\section{Pathological examination}

For pathological examination, the zebrafish were fixed in formalin and embedded in paraffin. Sections of $5 \mu \mathrm{m}$ were cut, deparaffinized, rehydrated, and stained with hematoxylin and eosin Y. A pathologist blinded to which experimental group the specimens were from evaluated the slides and reported the comparison results. ${ }^{18,19}$

\section{Statistics}

The Kaplan-Meier method was used to depict the curves for the cumulative survival rates of embryos in the control and LipoDox-injected groups. To assess the effect of drug on the increase of weight, we fitted the following linear regression model: weight $=\beta_{0}+\beta_{1}\{$ initial weight $\}+\beta_{2}\{$ time $\}+\beta_{3}\{$ group $\}+$ error; where $\beta_{0}$ is the intercept of the model, $\beta_{1}$ is the

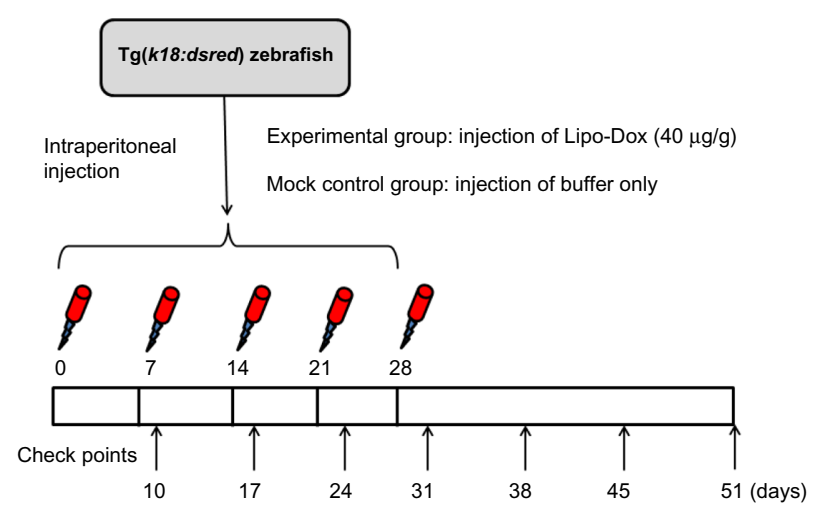

Figure I Schematic representation of experimental protocols performed in this study. Notes: $\operatorname{Tg}(k / 8: d s r e d)$ zebrafish were intraperitoneally injected either with buffer only (mock-control group, $\mathrm{n}=6$ ) or with buffer containing $40 \mu \mathrm{g} / \mathrm{g}$ of Lipo-Dox (Lipo-Dox ${ }^{\mathrm{TM}}$-injected group, $\mathrm{n}=12$, \#I-\#I2). We injected Lipo-Dox one dose (40 $\mu \mathrm{g} / \mathrm{g}$ ) at day 0 , and subsequently injected one dose per week for 4 weeks (days 7 , 14,21 , and 28 ), and examined the zebrafish phenotypic defects at each check point (days $10,17,24,31,38,45$, and 51 ). 
regression coefficient associated with initial weight (the weight at the beginning of the observation period, week 0 ), $\beta_{2}$ is the regression coefficient associated with time (the weeks after exposure to drug), and $\beta_{3}$ is the regression coefficient associated with group (group $=1$ for the drug group and 0 for the control group). Note that the measurements on the weight from the same embryo are correlated. To account for the within-embryo correlation, we applied the generalized estimating equation (GEE) analysis ${ }^{20}$ to estimate the parameters in the above model, assuming a normal distribution and a first-order autoregressive correlation structure for the data.

\section{Results}

\section{Gross assessment of HFS and intestinal mucositis in zebrafish model}

The results showed that the zebrafish receiving no Lipo-Dox (mock control) had a smooth belly and a regular-shaped caudal fin (Figure 2A-E). The Lipo-Dox-injected zebrafish had no apparent phenotypic changes at the first three check points (days 10,17, and 24), but intriguingly, displayed abdominal hemorrhage and fin fester, especially in the caudal fin regions at day 31 (Figure $2 \mathrm{~A}^{\prime}-\mathrm{E}^{\prime}$ ). Thus, the most obvious Lipo-Dox-induced phenotypes that can be observed grossly are abdominal hemorrhage and fin necrosis.

We observed fin necrosis and keratinocyte dissociation phenotypes in another two fish (\#5 and \#6) in the later stages (by days 38, 45, and 51) after discontinuation of Lipo-Dox administration (the latest injection was at day 28, Figure 1). Extensive fin necrosis at the caudal region was observed by day 38 , but gradually recovered by days 45 and 51 (Figure 3A-I versus B-I and C-I; Figure 3D-I versus E-I and F-I; indicated by arrows). Using fluorescent microscopy, we found that red fluorescent keratinocytes were aligned normally in the control zebrafish (data not shown), but
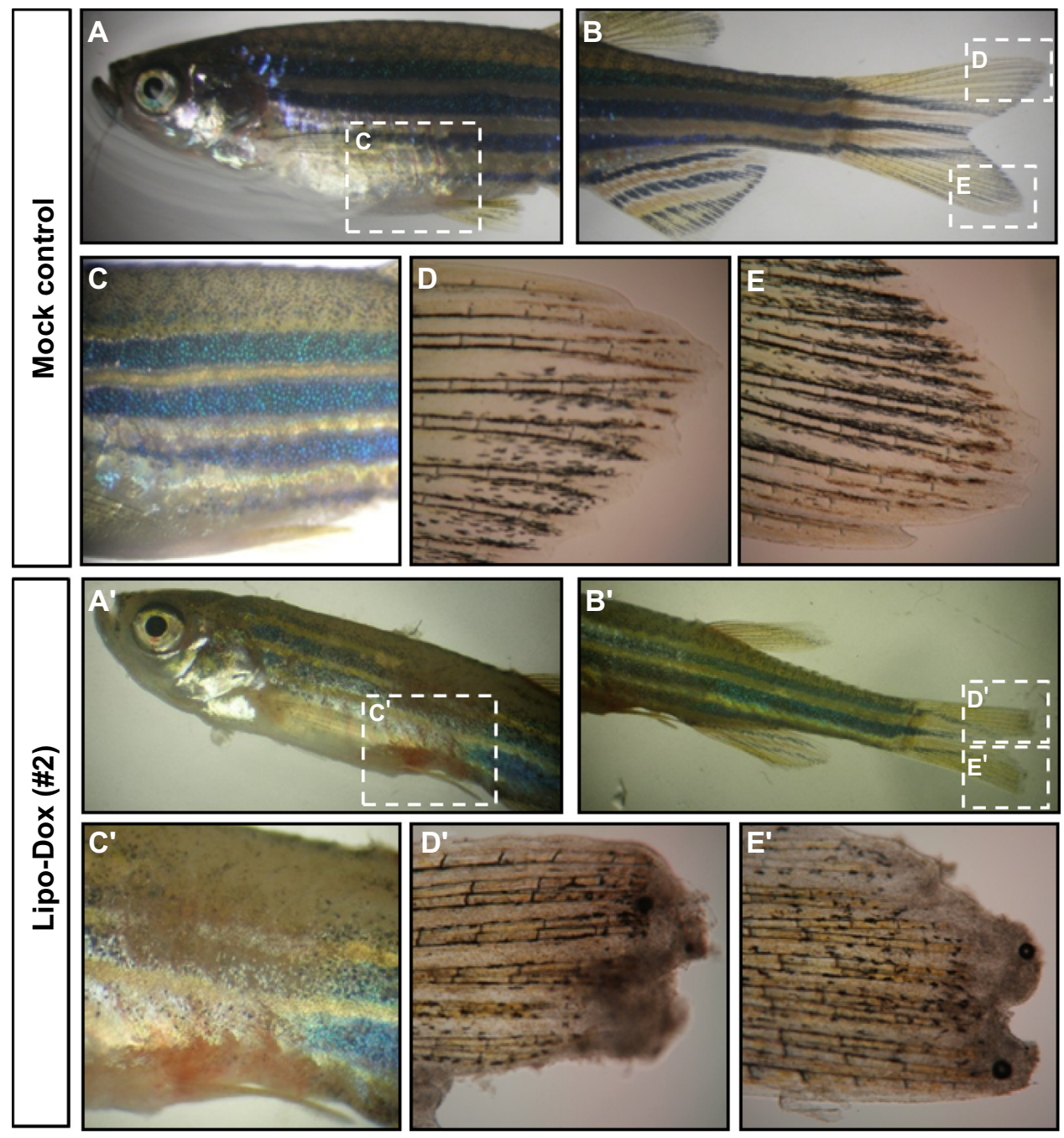

Figure 2 Abdominal hemorrhage and fin necrosis are observed in zebrafish embryos after Lipo-Dox ${ }^{\mathrm{TM}}$ injection.

Notes: (A-E) Mock control. (A'-E') Tg(k/8:dsred) zebrafish (\#2) was injected with buffer containing $40 \mu g / g$ of Lipo-Dox. 


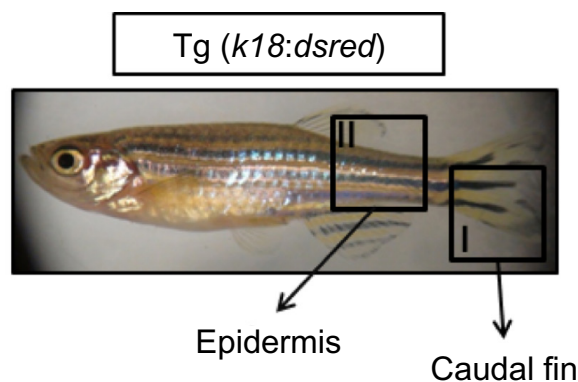

\section{Check points}

(days)

Lipo-Dox (\#5)
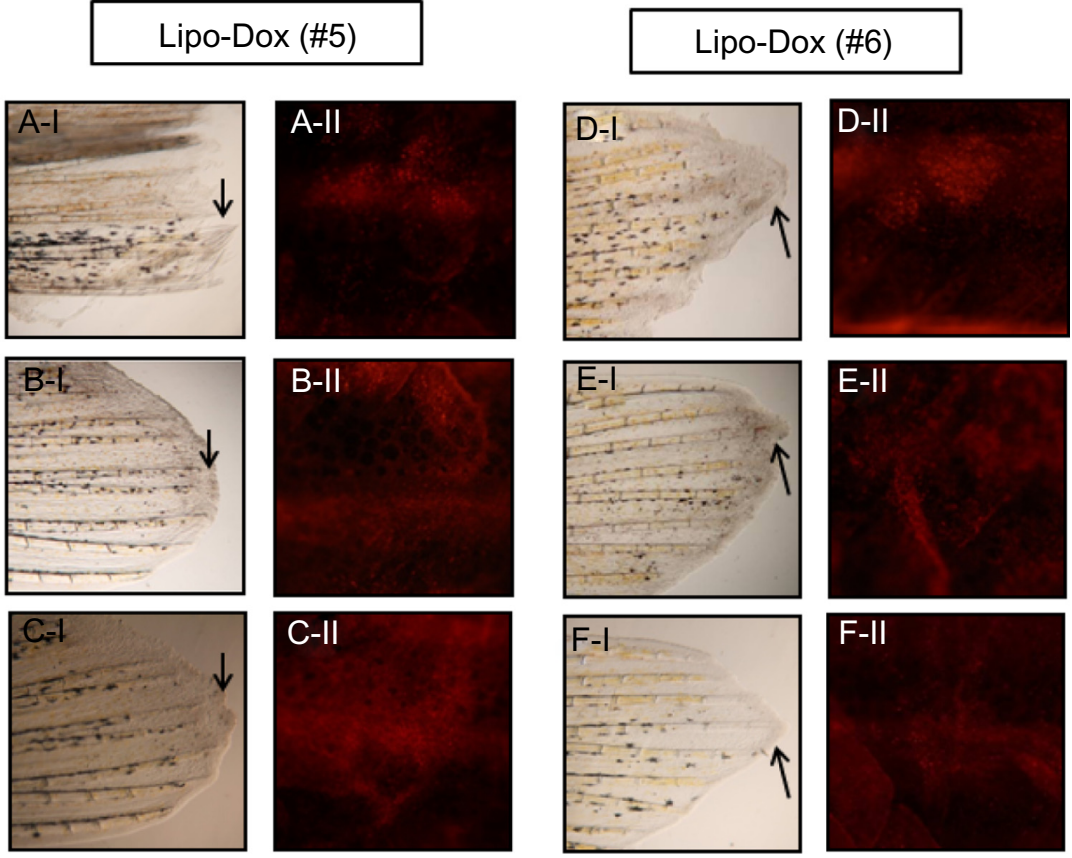

51

Figure 3 Effects of Lipo-Dox ${ }^{\mathrm{TM}}$ on the zebrafish's caudal fin (I) and epidermis (II).

Notes: Lipo-Dox-injected Tg(k/8:dsred) zebrafish (\#5 and \#6) were observed under microscopy with bright field (A-I-F-I) or an RFP filter (A-II-F-II). Fin necrosis (arrow indicates) and keratinocyte dissociation are the evident phenotypes in the Lipo-Dox-injected zebrafish.

Abbreviation: RFP, red fluorescent protein.
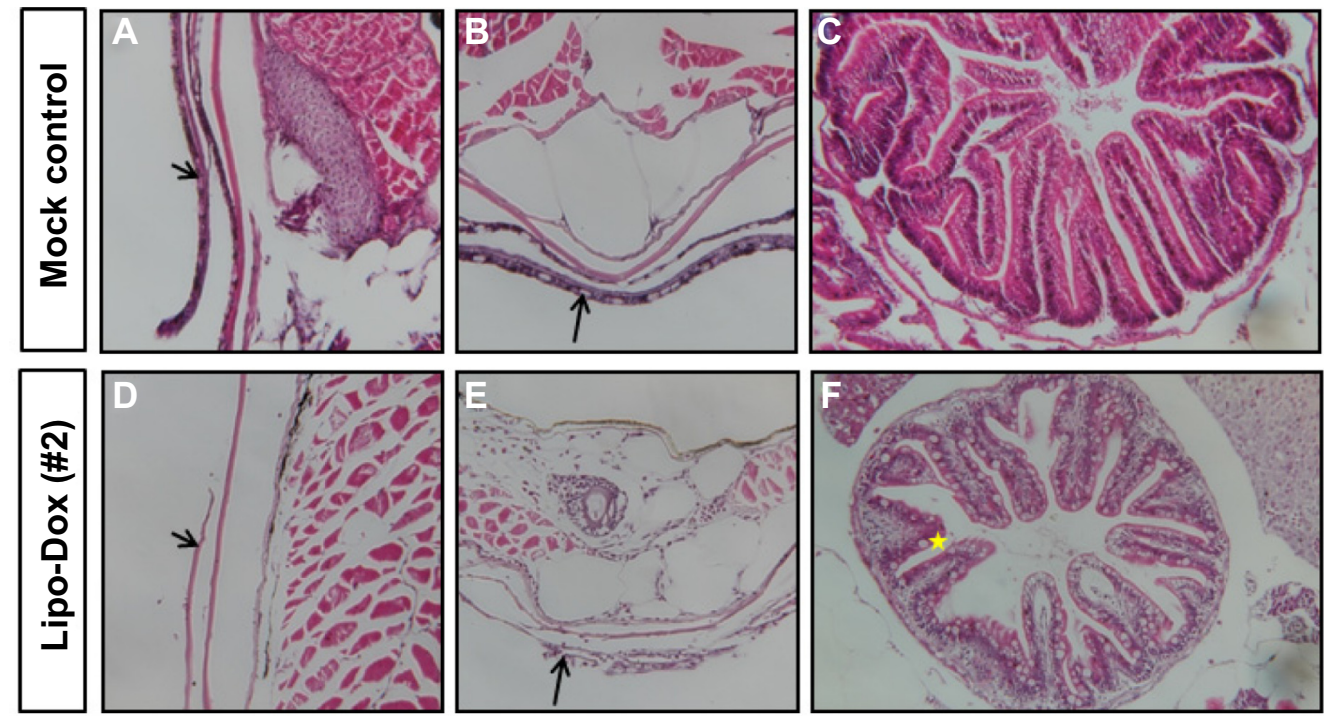

Figure 4 Histological examination of Lipo-Dox ${ }^{\mathrm{TM}}$-injected embryos.

Notes: Fish derived from the mock control $(\mathbf{A}-\mathbf{C})$ or Lipo-Dox-injected groups (D-F) were transverse sectioned and stained with hematoxylin/eosin Y. (A and $\mathbf{C})$ Lateral side; (B and E) Ventral region; (C and F) Intestine. Yellow star indicates the position of goblet cell. Black arrows indicate the positions of epidermis lesions. 
Table I Generalized linear regression based on the GEE method for assessing the effect of Lipo-Dox ${ }^{\mathrm{TM}}$ on the weight growth rates of embryos

\begin{tabular}{|c|c|c|c|c|c|c|}
\hline Variable & Estimate & $\begin{array}{l}\text { Standard } \\
\text { error }\end{array}$ & z-value & $P$-value & Lower CL & Upper CL \\
\hline Intercept & 0.037 & 0.043 & 0.877 & 0.381 & -0.046 & 0.120 \\
\hline Initial weight & 1.340 & 0.286 & 4.681 & $<0.001$ & 0.779 & 1.901 \\
\hline Group & -0.033 & 0.013 & -2.587 & 0.010 & -0.058 & -0.008 \\
\hline
\end{tabular}

Abbreviations: $\mathrm{CL}$, confidence level; GEE, generalized estimating equation.

appeared in a patch-like shape or even invisible on the surface (Figure 3A-II versus B-II). Those red fluorescent keratinocyte abnormalities gradually recovered by days 45 and 51 (Figure 3C-II, D-II, E-II, and F-II), compatible with the gross finding.

\section{Pathological examination of abdominal skin and intestines}

We next carried out paraffin sectioning and hematoxylin/ eosin Y staining experiments to further dissect the Lipo-Dox-induced abdominal hemorrhage phenotypes from the histological level. The results showed that several mucosa cells appeared in the skin epidermis, but very few goblet cells were observed in the intestine region in the mocktreated control zebrafish (Figure 4A-C). However, missing mucosa cells with impaired mucosal integrity, thinner skin epidermis, and increased numbers of goblet cells (a marker for intestine inflammatory reaction) were observed in the Lipo-Dox-injected zebrafish (Figure 4D-F).

\section{Growth retardation of zebrafish}

We noticed that the Lipo-Dox-injected zebrafish larvae exhibited various degrees of growth retardation and impaired survival. For growth retardation, Table 1 displays the analysis result by GEE model. It shows that the growth, estimated by weight, in the Lipo-Dox-injected group was significantly less than the control group $(P=0.010)$. The embryos in the Lipo-Dox-injected group tended to have a lower weight of $0.033 \mathrm{~g}$ (with a standard error of $0.013 \mathrm{~g}$ ) than those in the control group, adjusting the time after exposure to Lipo-Dox and the initial weight by the GEE method.

\section{Survival analysis of zebrafish}

We first applied the Kaplan-Meier method to depict the survival curves of embryos in the control and Lipo-Doxinjected groups. As shown in Figure 5, a 25\% death rate at the end of study, 7.43 weeks after exposure to drug, in the Lipo-Dox-injected group was noted. The mean survival time for the Lipo-Dox-injected group was $5.26 \pm 0.13$ weeks.

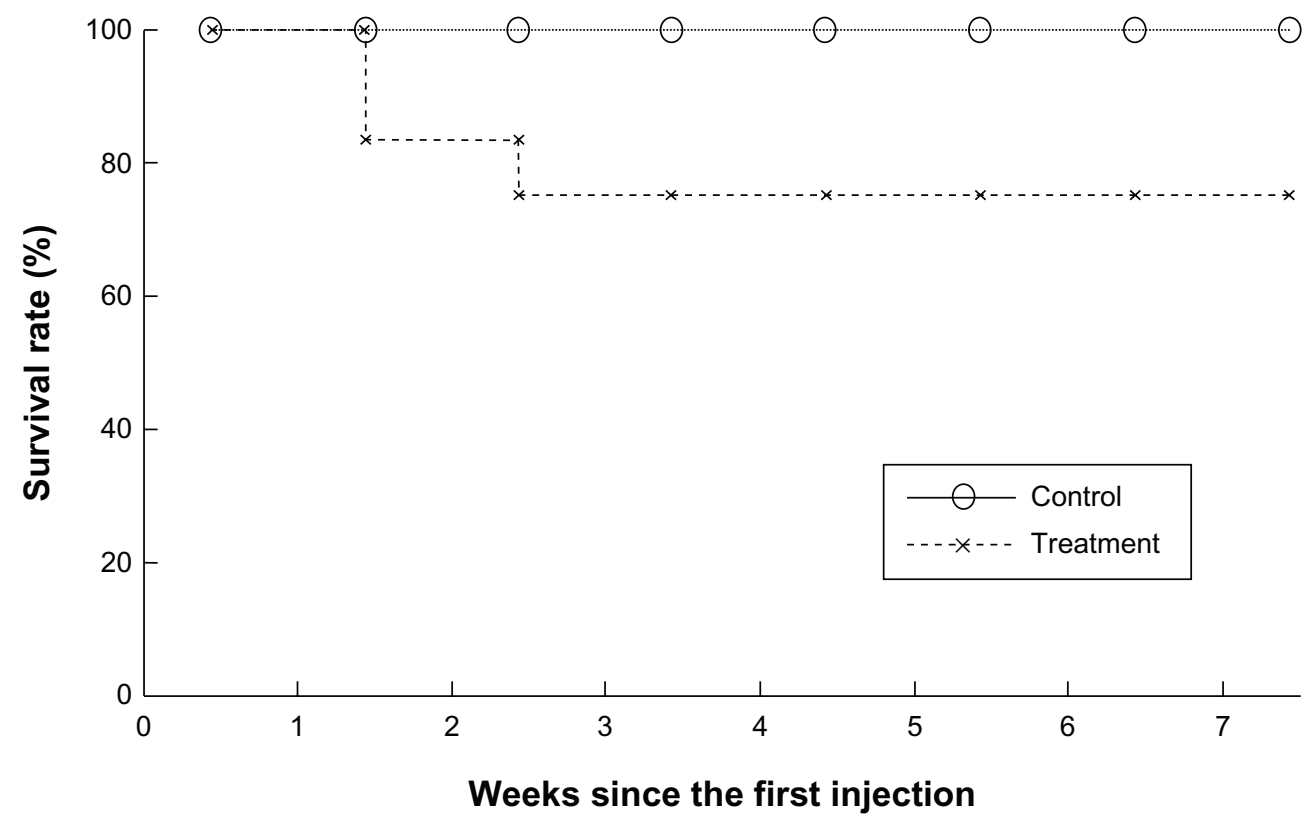

Figure 5 Kaplan-Meier estimates of survival curves for the mock control and Lipo-Dox ${ }^{\mathrm{TM}}{ }_{\text {-injected groups. }}$ 
No embryos died in the control group. The log-rank test was further utilized to examine the homogeneity of the death rate curves for the two groups. The result shows a significant difference in time to death between the groups $(P$-value $=0.043)$, confirming the Lipo-Dox-injected group had suffered a significant harmful effect.

\section{Conclusion}

In the zebrafish model we established for gross assessment of PLD-induced HFS, we observed both the intestinal mucositis, a common side effect of PLD in humans, as well as skin damage with the typical pathological changes of HFS, validating that this model is clinically feasible due to the similarities in the clinical course and manifestations. The toxicity induced by Lipo-Dox injection was reversible, as is the case in clinical practice, where the typical skin pathological changes of HFS disappear within a few weeks after discontinuation of the drug. ${ }^{12}$

The markedly increased number of intestinal goblet cells (an important marker of intestinal inflammation) $)^{21,22}$ we observed in the Lipo-Dox-injected zebrafish suggest that the intestinal inflammation induced by Lipo-Dox resembles the intestinal mucositis the clinical patients suffered from after the administration of this anticancer therapeutic.

Our data suggest that the zebrafish can be regarded as an efficient screening model for agents managing PLD-induced HFS, as well as intestinal mucositis. However, further clinical investigations to validate the correlation between human and zebrafish data are warranted.

\section{Acknowledgments}

We are grateful to Professor Huei-Jen Tsai (Institute of Molecular and Cell Biology, National Taiwan University, Taipei, Taiwan) who provided the $\operatorname{Tg}(k 18: d s r e d)$ fish. This work was supported by grants MMH-E-102-13.

\section{Disclosure}

The authors do not have and have not had a relationship with the manufacturers of the materials involved, nor have they received funding from the manufacturers to carry out their research. The authors claim they have no conflict of interest in this work.

\section{References}

1. Ferrandina G, Ludovisi M, Lorusso D, et al. Phase III trial of gemcitabine compared with pegylated liposomal doxorubicin in progressive or recurrent ovarian cancer. J Clin Oncol. 2008;26(6):890-896.

2. Alba E, Ruiz-Borrego M, Margeli M, et al. Maintenance treatment with pegylated liposomal doxorubicin versus observation following induction chemotherapy for metastatic breast cancer: GEICAM 2001-01 study. Breast Cancer Res Treat. 2010;122(1):169-176.
3. Goebel FD, Goldstein D, Goos M, Jablonowski H, Stewart JS. Efficacy and safety of stealth liposomal doxorubicin in AIDS-related Kaposi's sarcoma. The International SL-DOX Study Group. $\mathrm{Br} J$ Cancer. 1996;73(8):989-994.

4. Pujade-Lauraine E, Wagner U, Aavall-Lundqvist E, et al. Pegylated liposomal doxorubicin and carboplatin compared with paclitaxel and carboplatin for patients with platinum-sensitive ovarian cancer in late relapse. J Clin Oncol. 2010;28(20):3323-3329.

5. Hong RL, Huang CJ, Tseng YL, et al. Direct comparison of liposomal doxorubicin with or without polyethylene glycol coating in C-26 tumorbearing mice: is surface coating with polyethylene glycol beneficial? Clin Cancer Res. 1999;5(11):3645-3652.

6. Coukell AJ, Spencer CM. Polyethylene glycol-liposomal doxorubicin. A review of its pharmacodynamic and pharmacokinetic properties, and therapeutic efficacy in the management of AIDS-related Kaposi's sarcoma. Drugs. 1997;53(3):520-538.

7. O'Brien ME, Wigler N, Inbar M, et al. Reduced cardiotoxicity and comparable efficacy in a phase III trial of pegylated liposomal doxorubicin $\mathrm{HCl}$ (CAELYX/Doxil) versus conventional doxorubicin for first-line treatment of metastatic breast cancer. Ann Oncol. 2004;15(3): 440-449.

8. Chao TC, Wang WS, Yen CC, et al. A dose-escalating pilot study of sterically stabilized, pegylated liposomal doxorubicin (Lipo-Dox) in patients with metastatic breast cancer. Cancer Invest. 2003;21(6):837-847.

9. Kaczmarek A, Brinkman BM, Heyndrickx L, Vandenabeele P, Krysko DV. Severity of doxorubicin-induced small intestinal mucositis is regulated by the TLR-2 and TLR-9 pathways. J Pathol. 2012;226(4): 598-608.

10. Khemapech N, Oranratanaphan S, Termrungruanglert W, Lertkhachonsuk R, Vasurattana A. Salvage chemotherapy in recurrent platinum-resistant or refractory epithelial ovarian cancer with carboplatin and distearoylphosphatidylcholine pegylated liposomal doxorubicin (Lipo-Dox(R)). Asian Pac J Cancer Prev. 2013;14(3):2131-2135.

11. Lacouture ME, Reilly LM, Gerami P, Guitart J. Hand foot skin reaction in cancer patients treated with the multikinase inhibitors sorafenib and sunitinib. Ann Oncol. 2008;19(11):1955-1961.

12. Baack BR, Burgdorf WH. Chemotherapy-induced acral erythema. J Am Acad Dermatol. 1991;24(3):457-461.

13. Baker TR, Peterson RE, Heideman W. Using zebrafish as a model system for studying the transgenerational effects of dioxin. Toxicol Sci. 2014;138(2):403-411.

14. Lele Z, Krone PH. The zebrafish as a model system in developmental, toxicological and transgenic research. Biotechnol Adv. 1996; 14(1):57-72.

15. Ding YJ, Chen YH. Developmental nephrotoxicity of aristolochic acid in a zebrafish model. Toxicol Appl Pharmacol. 2012;261(1):59-65.

16. Wang YH, Chen YH, Lin YJ, Tsai HJ. Spatiotemporal expression of zebrafish keratin 18 during early embryogenesis and the establishment of a keratin 18:RFP transgenic line. Gene Expr Patterns. 2006;6(4):335-339.

17. Wang YH, Chen YH, Wu TN, Lin YJ, Tsai HJ. A keratin 18 transgenic zebrafish $\operatorname{Tg}(\mathrm{k} 18(2.9)$ :RFP) treated with inorganic arsenite reveals visible overproliferation of epithelial cells. Toxicol Lett. 2006;163(3): 191-197.

18. Pai CW, Chen YH. Transgenic expression of prothymosin alpha on zebrafish epidermal cells promotes proliferation and attenuates UVB-induced apoptosis. Transgenic Res. 2010;19(4):655-665.

19. Stuart GW, Vielkind JR, McMurray JV, Westerfield M. Stable lines of transgenic zebrafish exhibit reproducible patterns of transgene expression. Development. 1990;109(3):577-584.

20. Shults J, Mazurick CA, Richard Landis J. Analysis of repeated bouts of measurements in the framework of generalized estimating equations. Stat Med. 2006;25(23):4114-4128.

21. Sommers SC, Korelitz BI. Mucosal-cell counts in ulcerative and granulomatous colitis. Am J Clin Pathol. 1975;63(3):359-365.

22. Norris AA, Lewis AJ, Zeitlin IJ. Changes in colonic tissue levels of inflammatory mediators in a guinea-pig model of immune colitis. Agents Actions. 1982;12(1-2):243-246. 
OncoTargets and Therapy

\section{Publish your work in this journal}

OncoTargets and Therapy is an international, peer-reviewed, open access journal focusing on the pathological basis of all cancers, potential targets for therapy and treatment protocols employed to improve the management of cancer patients. The journal also focuses on the impact of management programs and new therapeutic agents and protocols on

patient perspectives such as quality of life, adherence and satisfaction. The manuscript management system is completely online and includes a very quick and fair peer-review system, which is all easy to use. Visit http://www.dovepress.com/testimonials.php to read real quotes from published authors.

Submit your manuscript here: http://www.dovepress.com/oncotargets-and-therapy-journal 